\title{
Some remarks on the influence of temperature-variations, non- linearities, repeatability and ageing on modal-analysis for structural health monitoring of real bridges
}

\author{
Stefan Maas ${ }^{1 a}$, Sebastian Schommer ${ }^{1}$, Viet-Hà Nguyen ${ }^{1}$, Danièle Waldmann ${ }^{1}$, Jean Mahowald ${ }^{1}$, Arno Zürbes ${ }^{2}$ \\ ${ }^{1}$ University of Luxembourg, Faculty of Science, Technology and Communication (FSTC), Campus Kirchberg, \\ Research Unit of Engineering Sciences (RUES), L-1359 Luxembourg \\ ${ }^{2}$ Fachhochschule D-55411 Bingen, Germany
}

\begin{abstract}
Structural Health Monitoring (SHM) intends to identify damage by changes of characteristics as for instance the modal parameters. The eigenfrequencies, mode-shapes and damping-values are either directly used as damage indicators or the changes of derived parameters are analysed, such as e.g. flexibilities or updated finite element models. One common way is a continuous monitoring under environmental excitation forces, such as wind or traffic, i.e. the so-called output-only modal analysis. Alternatively, a forced measured external excitation in distinct time-intervals may be used for input-output modal analysis. Both methods are limited by the precision or the repeatability under real-life conditions at site. The paper will summarize several field tests of artificially stepby-step damaged bridges prior to their final demolishment and it will show the changes of eigenfrequencies due to induced artificial damage. Additionally, some results of a monitoring campaign of a healthy bridge in Luxembourg are presented. Reinforced concrete shows non-linear behaviour in the sense that modal parameters depend on the excitation force amplitude, i.e. higher forces lead often to lower eigenfrequencies than smaller forces. Furthermore, the temperature of real bridges is neither constant in space nor in time, while for instance the stiffness of asphalt is strongly dependant on it. Finally, ageing as such can also change a bridge's stiffness and its modal parameters, e.g. because creep and shrinkage of concrete or ageing of elastomeric bearing pads influence their modulus of elasticity. These effects cannot be considered as damage, though they influence the measurement of modal parameters and hinder damage detection.
\end{abstract}

\section{Introduction}

Damage due to corrosion and fatigue of a bridge leads to reduction of its stiffness and in a smaller extend also to changes in the damping behaviour. Hence, the modal parameters change, i.e. the eigenfrequencies, modeshapes, damping-values. Structural Health Monitoring (SHM) intends to detect damage by measurement and analysis of changes of characteristics leading to the changes in the stiffness matrix. These changes can be identified in multiple ways, but very often the modal parameters are the starting point (ref. for example to J. Mahowald [1]).

This monitoring and surveying of a bridge is either done continuously and automatically or in discrete timeintervals. It may be input and output or output-only measurements. Additionally, the evaluation can be model-based or not. However, most methods try to reveal changes of modal parameters or deduced characteristics to compare them to a healthy reference state in order to reveal stiffness changes, i.e. damage.

Unfortunately the measured parameters are not only influenced by damage, but also by a) temperature, b) the magnitude of excitation-force and c) ageing process of concrete and d) uncertainties and random noise.

Subsequently we will present the above-mentioned interference effects and deploy that their impact on the dynamic characteristics is in the same order of magnitude than damage. We will show experiments on real bridges, where artificial damage was successively introduced in multiple steps prior to their demolishment. Furthermore, we will analyse output-only monitoring results of a new small and healthy two span composite bridge in Useldange Luxembourg that we follow-up for approximately 10 years.

\section{Non-linearities and parameter variations}

Cracks of concrete show different stiffness behaviour when they open or close and any concrete comprises at least mirco-cracks. Hence, it is not astonishing to find as well on test beams in the laboratory as well on real bridges non-linearities. To study these effects we choose swept-sine excitation with constant force amplitude, i.e. the excitation force amplitude was kept constant while slowly changing the harmonic excitation frequency with

\footnotetext{
a Corresponding author: stefan.maas@uni.lu
} 
typically $1 \mathrm{~Hz}$ per minute. In any case the excitation forces (input) were measured and their amplitude was kept constant. The measured force was used together with the accelerations of the bridge (output) to determine the frequency-response-functions (FRF). Figure 1 and 2 show examples of a test beam of reinforced concrete without prestress in our lab [2] and of a real bridge in Useldange [3]. With increasing excitation force we find decreasing stiffness and hence decreasing resonance frequencies, which is a non-linearity and which has an impact of $2 \%$ or even $3 \%$ on the measured resonance frequency. J. Mahowald confirmed this order of magnitude of some percent for the excitation force nonlinearity on a second real bridge, the so-called Champangshiel-Bridge in Luxembourg [1].

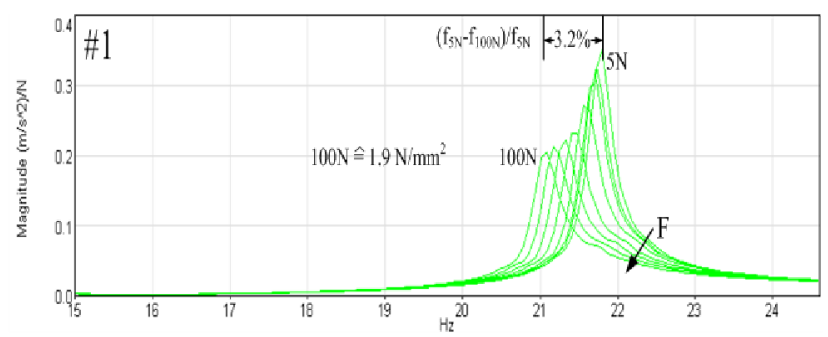

Figure 1. FRFs of a reinforced test beam excited with 8 different force amplitudes between $5 \mathrm{~N}$ and $100 \mathrm{~N}$ leading to a variation of $3.2 \%$ for the eigenfrequency [2].

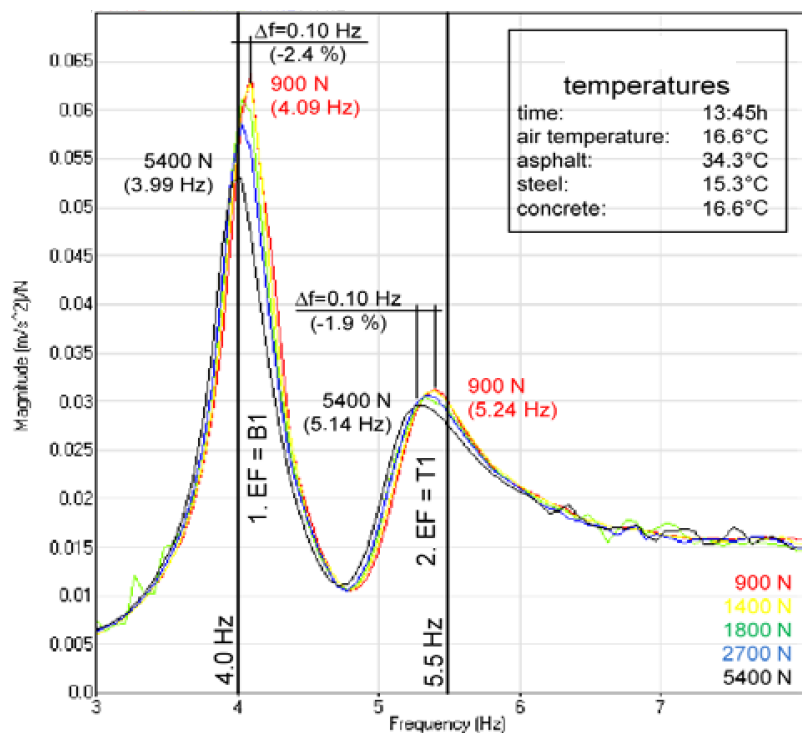

Figure 2. FRFs of Useldange Bridge excited with five different force amplitudes between $900 \mathrm{~N}$ and $5400 \mathrm{~N}$ leading to a variation of $2.4 \%$ for the eigenfrequency [3].

Special mobile exciters were designed, built and used for the bridge tests (Figure 3), while in the laboratory an electrodynamic shaker was used for forces below $2.7 \mathrm{kN}$.
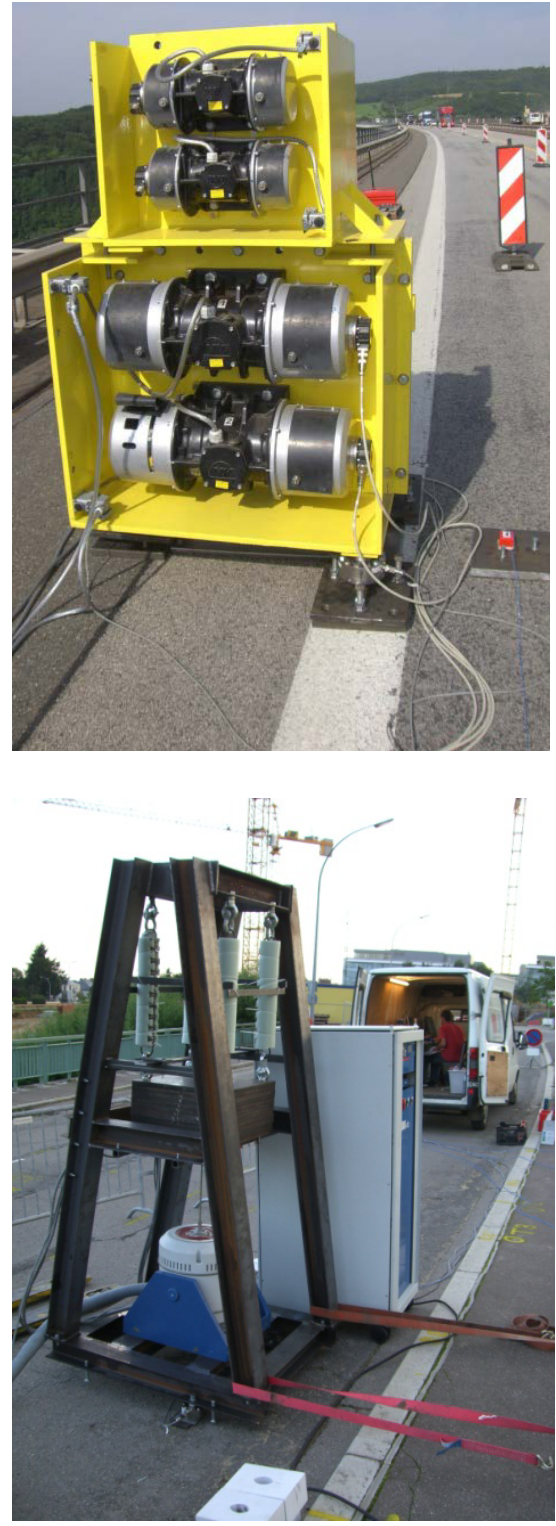

Figure 3. Harmonic force exciters mounted of force-cells for swept-sine excitation: Unbalanced mass exciter (top) and electromagnetic shaker (bottom).

With the help of these exciters it is possible generate the necessary forces and to keep the excitation force constant. A more detailed description of these exciters can be found in [4]. If the excitation force is not controlled e.g. when hammer impact or environmental forces such as wind or traffic are used to excite the bridge, some percent of uncertainty have to be considered when analysing the results as this effect will reduce the repeatability of the measurements. Especially when we want to monitor changes over years, we should keep the excitation forces constant. So, when environmental excitation (wind \& traffic) is used for modal analysis, only extensive averaging can attenuate these effects while creating timedelay.

Needless to say that also a modified mass loading of a bridge due to traffic or replaced asphalt layer can also change the eigenfrequencies. 


\section{Temperature effects}

It is known that the stiffness of asphalt for instance is strongly dependent on temperature. Young's modulus of asphalt is varying approximately between $500 \mathrm{MPa}$ at $40^{\circ} \mathrm{C}$ and $20000 \mathrm{MPa}$ at $-10^{\circ} \mathrm{C}$ and furthermore dependent on the strain-rate, which is a non-linear phenomenon [5]. Elastomeric bearing pads for bridges and also the subsoil show temperature and preload dependant characteristics. Hence, it is not astonishing that temperature has an important influence on the eigenfrequencies [6]. Figure 4 shows monitoring results of two different bridges with two very different slopes and bandwidths, which depend on the bridge's asphalt layer, the construction type and the foundations. So, the slope and the bandwidth are individual characteristics of a bridge, which cannot be ignored, as both might have big impact. For example, for the Useldange bridge we find a slope of $7 \%$ per ${ }^{\circ} \mathrm{C}$ and for the Z24 only $1 \%$ o per ${ }^{\circ} \mathrm{C}$. This slope finally has to be used for temperature effect compensation, while the bandwidth is an unpleasant uncertainty (e.g. $13 \%$ in Useldange and $2.5 \%$ for Z24) and influenced by different factors, for instance varying mass-loading (traffic), nonlinearities and other parameters, which will be subsequently discussed.

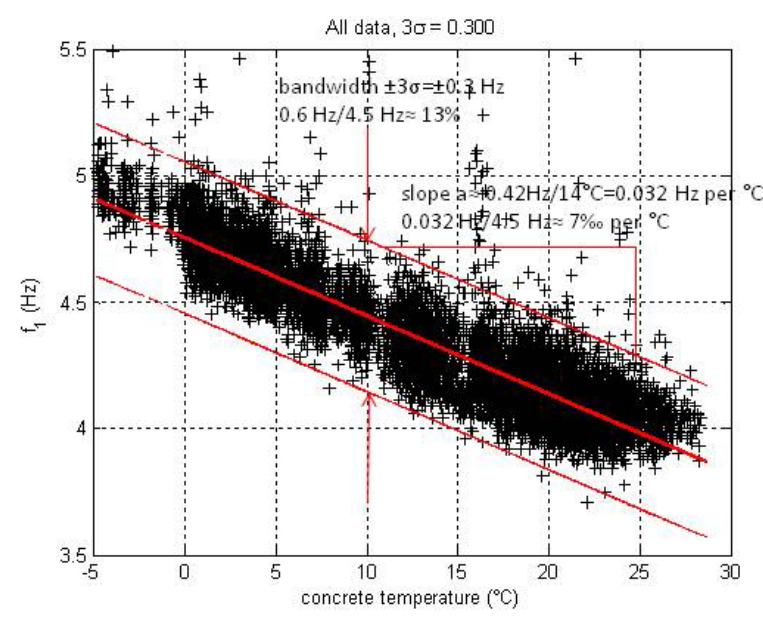

4a. Bridge in Useldange (L) (2008) [6].

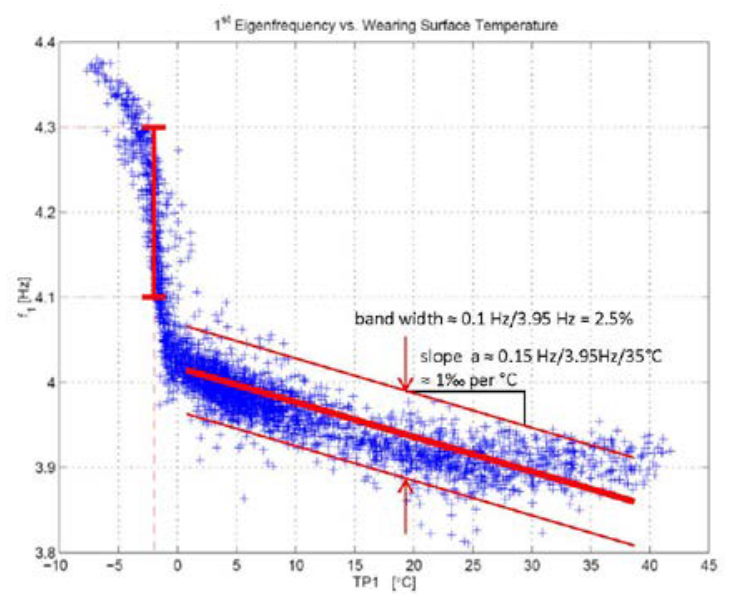

4b. Bridge Z24 (CH) (1997-1998) [7].

Figure 4. First eigenfrequency versus the temperature for different bridges.
Looking more in detail into the temperature dependency one quickly finds out, that normally a bridge has not "a" temperature for a given moment in time, but that temperature varies from point to point, i.e. temperature is a scalar field for the bridge in space and time $\mathrm{T}=\mathrm{T}(\mathrm{x}, \mathrm{y}, \mathrm{z}, \mathrm{t})$. This is especially true for sunny days, as normally the solar radiation is not constantly distributed. The Useldange bridge in Luxembourg is a composite two-span bridge. The upper plate has a thickness of $25 \mathrm{~cm}$ and is made of concrete $\mathrm{C} 45 / 55$ and covered by the asphalt layer. This concrete plate is held by four main longitudinal steel girders of S355, i.e. the bridge is a mixed construction. In Figure 5 we show 4 temperatures measured inside the concrete plate (in red) and 4 temperatures measured externally on the steel girders (in blue) below the deck. The steel temperatures follow more or less the air temperatures with a period of 24 hours. As during midnight the data acquisition system was shut down for some hours to save memory the lines are not continuous. The eigenfrequencies (in green) were identified by stochastic subspace identification (SSI) and clearly also show the sinusoidal temperature variation.

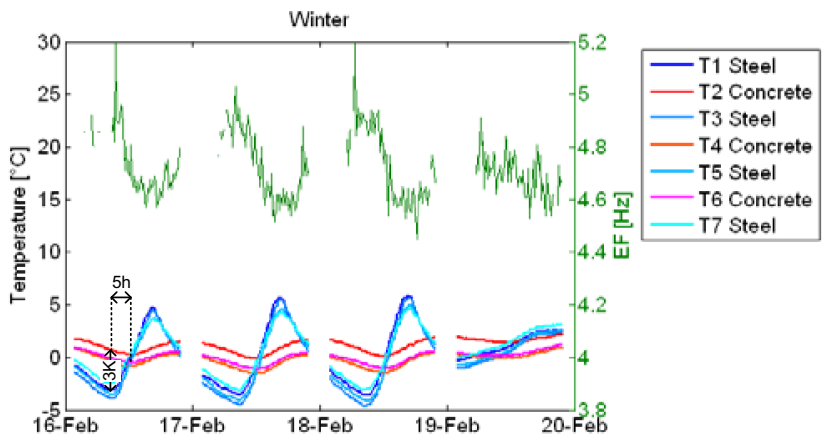

Figure 5. Temperatures and eigenfrequencies of Useldange bridge in Winter 2008 for subsequent sunny days [1]..

On cloudy days (ref. to Figure 6) we see that the temperatures are close together and hence the eigenfrequencies are quite constant. These cloudy days should be used for measurements, as we can attribute more or less "one" temperature to the complete bridge.

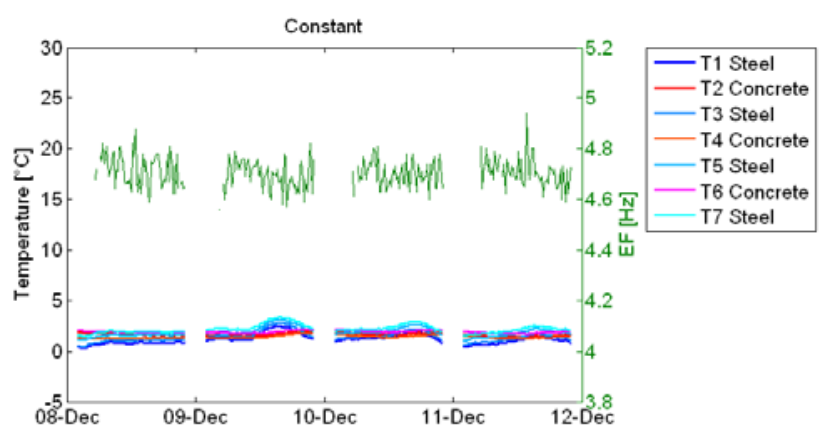

Figure 6. Temperatures and eigenfrequencies of Useldange bridge in Winter 2008 for some cloudy days [1].

A deeper analysis of these data was done in [6] and revealed that on sunny days even the temperatures differences between steel and concrete have an influence on the eigenfrequencies, so that these days cannot be 
recommended for modal identification. A temperature compensation of the measured eigenfrequencies is mandatory prior to final assessment of the bridge and can be done, when the individual slope of this bridge (ref. to Figure 4) has been determined.

\section{4 "Normal" ageing}

It is well known that concrete for instance is subject to shrinkage and creep, which changes its properties before all in the first months. But even after years, e.g. its apparent Young's modulus is affected and modified. These effects cannot be considered as damage, but just as "normal" ageing (ref. e.g. to [8], [9], [10], [11]). The variations have influence on the dynamic monitoring in the range of some percent on the eigenfrequencies. Figure 7 shows the results for the lowest three eigenfrequences for Useldange Bridge evaluated for the years 2007-2010 by output-only measurements. The Stochastic SubspaceIdentification (SSI) algorithm (ref. to [12],[13],[1]) was used, but only for temperature differences of steel and concrete temperatures of $\Delta \mathrm{T} \leq \pm 1.5^{\circ} \mathrm{C}$.

\section{$\mathrm{f} 1[\mathrm{~Hz}]$}

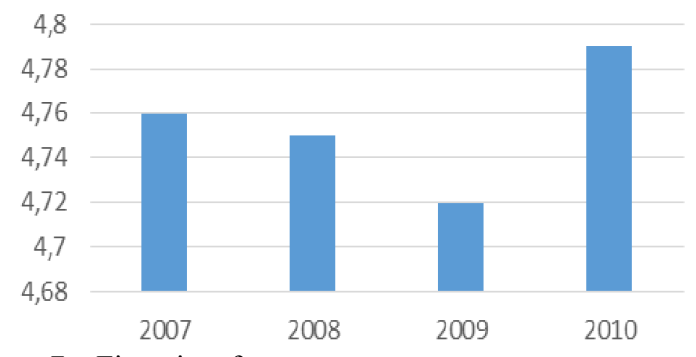

7a. First eigenfrequency.

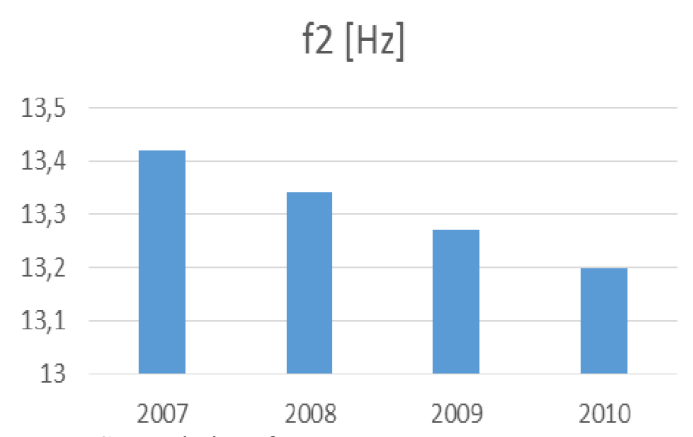

7b. Second eigenfrequency.

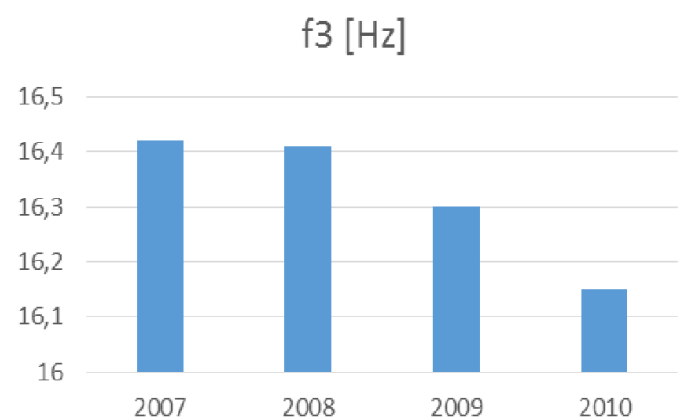

7c. Third eigenfrequency.

Figure 7. Variations of the eigenfrequencies of Useldange Bridge from 2007-2010.
Figure 7 reveals changes up to $2 \%$ due to normal ageing.

\section{Repeatability and damage}

Even if we keep the excitation force and the temperatures constant within the bounds of possibility, we faced on real bridges problems with the repeatability. Two successive measurements within 30 minutes showed differences up to $1 \%$ for the first eigenfrequency, though we used our exciters with constant excitation force amplitude shown in Figure 3.

The Champangshiehl bridge in Luxembourg was artificial damaged by cutting tendons in four successively damage scenarios, hereafter denoted as scenario \#0 (undamaged) to scenario \#4 (approximately half of the tendons locally cut). An exhaustive description of the bridge and the tests can be found in J. Mahowald [1]. The eigenfrequencies of this two span prestressed concrete girder box bridge were identified in four successive damage scenarios, i.e. more and more tendons were cut from scenario $\# 0$ to $\# 4$. In Figure 8 the measured eigenfrequencies of the first bending mode (B1) and the second torsion mode (T2) are depicted. If tendons are cut, the prestress is at least locally reduced leading to a decrease of the eigenfrequencies in any theoretical model, while we found partly an increase of up to $7.2 \%$ for torsion mode $\mathrm{T} 2$, which can only be explained by errors and perhaps temperature effects though there was no asphalt layer on this bridge and all tests were done within one month.

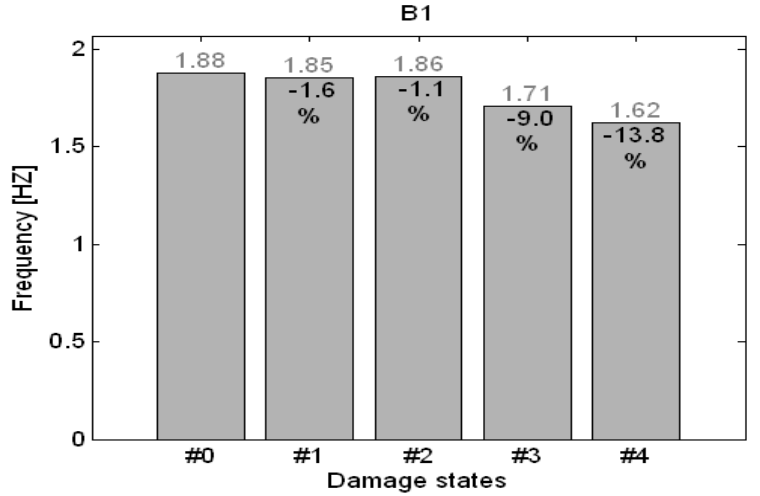

8a. First bending eigenfrequency (B1).

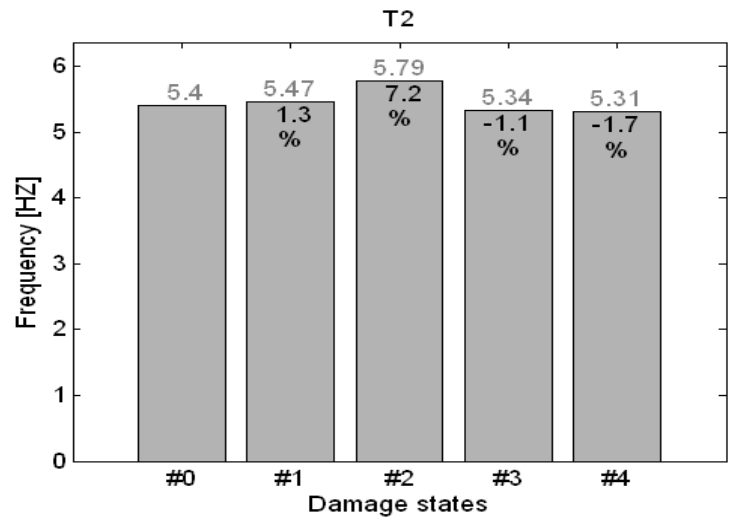

8b. Second torsion eigenfrequency (T2).

Figure 8. Measured eigenfrequencies for two modes of Champangshiel bridge for five different damage scenarios \#0 to \#4 [1]. 
A promising statistical approach to cope with these environmental effects seems Principal Component Analysis (PCA) as deployed by V.H. Nguyen et al. in [14].

Furthermore, we see in Figure 8 that the effect of severe damage changes the eigenfrequencies only by approximately $15 \%$, as in the last scenario \#4 half of the tendons were cut at two locations and we detected visible cracks in the prestressed concrete.

This reveals the core of the problem: the uncertainties due to non-linearities, due to temperature and due to measuring errors are in the same order of magnitude that the influence of severe damage.

\section{Summary and Conclusions}

Multiple techniques of Structural Health Monitoring (SHM) are based on modal parameter identification. The eigenfrequencies are more sensitive with respect to damage than modeshapes, damping values and modal masses [2], [3], [4]. The full set of modal parameters can be used for instance to determine the flexibility or even the stiffness-matrix and hence reveal changes due to damage, provided their accuracy is sufficient [6]. Unfortunately, it is shown that on real bridges nonlinearities, random-measurement noise, normal ageing and before all temperature effects truncate the modal parameters in the same order of magnitude than severe damage, resulting in problems for damage identification and localisation.

We propose to use swept sine excitation to generate well measurable, repeatable harmonic excitation and to keep mass loading constant, i.e. measure without traffic. Concerning the temperature influence, we suggest to do repetitive measurements on cloudy days with different temperatures until the dependency of the eigenfrequencies with respect to the temperature is known. An output-only monitoring for some weeks or months can also help to determine the slope of the eigenfrequencies with respect to temperature, as shown for instance in Figure 4. With the help of this characteristic slope we can calculate all eigenfrequencies for a reference temperature, e.g. $10^{\circ} \mathrm{C}$ the approximate mean annual temperature.

Further research has to be done to improve the repeatability and/or the assessment of the measurements. Principal Component Analysis seems to be a promising way to reduce the overlaid measurement noise [14].

\section{References}

1. J. Mahowald, Evaluation of Dynamic Damage Indicators on real-life civil engineering structures: measurement uncertainty and environmental influences considered, Dissertation PhD-FSTC2013-11 University of Luxembourg, Shaker Verlag GmbH, Germany, ISBN 978-3-8440-2673-3

2. M. Waltering, Damage Assessment of Civil Engineering Structures and Bridges Using Nonlinear Dynamic Characteristics, Dissertation PhD-FSTC-1-
2009, University of Luxembourg, Shaker Verlag GmbH, Germany, ISBN 978-3-8322-8611-8

3. V. Bungard, Condition Assessment of concrete structures and bridges using vibration monitoring in comparison to changes in their static properties, Dissertation PhD-FSTC-26-2010, University of Luxembourg, Shaker Verlag GmbH, Germany, ISBN 978-3-8440-0077-1

4. S. Maas et al., Damage assessment of concrete structures through dynamic testing methods. Part 2: Bridge tests, Elsevier, Engineering Structures 34 (2012) 483-494

5. R. Blab, Asphalt im Kontext der neuen Europäischen Normen, Gestrata Journal, Folge 112, pp.11-18, (2006)

6. J. Mahowald, S. Maas, V.H. Nguyen, D. Waldmann, A. Zürbes, Some conclusions from the measurements of temperatures and their gradients on eigenfrequencies of bridges, Proceedings of $9^{\text {th }}$ Eurodyn 2014, Porto, Portugal

7. B. Peeters, G. De Roeck, One Year Monitoring of the $z$ 24-bridge: Environmental Influences Versus Damage Events, Proceedings of the international Modal Analysis Conference IMAC 2000, p. 1570 1576

8. F. Magalhães, A. Cunha, E. Caetano, Five years of continuous dynamic monitoring of Infante $D$. Henrique Bridge, Proceedings of the 9th International Conference on Structural Dynamics, EURODYN 2014, Porto, Portugal

9. Gomez Hugo C., Fanning Paul, Feng Maria Q., Lee Sungchil, Testing and long-term monitoring of $a$ curved concrete box girder bridge, Engineering Structures, 33 (2010), p. 2861-2869

10. Soyoz S., Maria Q. Feng, Long-Term Monitoring and Identification of Bridge Structural Parameters, Computer-Aided Civil and Infrastructure Engineering, 11/2008, 24(2), p. 82 - 92

11. Au Francis T.K. and Si X.T., Time-dependent effects on dynamic properties of cable-stayed bridges, Structural Engineering and Mechanics, Vol. 41, No. 1, 2012, p. 139-155

12. A. Deraemaeker, E. Reynders, G. De Roeck, and J. Kullaa, Vibration-based Structural Health Monitoring Using Output-only Measurements Under Changing Environment, Mechanical Systems and Signal Processing 22 (1) (January 2008): p. 34-56. doi:10.1016/j.ymssp.2007.07.004

13. P. van Overschee and Bart L. R. de Moor, Subspace Identification for Linear Systems: Theory, Implementation, Applications, Kluwer Academic Publishers (1996)

14. V.H. Nguyen, J. Mahowald, J.C. Golinval, S. Maas, Damage detection in bridge structures including environmental effects, Proceedings of $9^{\text {th }}$ Eurodyn 2014, Porto, Portugal 